\title{
SALUD MENTAL Y SU RELACION CON LAS CARACTERISTICAS BIOSOCIODEMOGRAFICAS EN PACIENTES HEMODIALIZADOS
}

\author{
MENTAL HEALTH AND ITS RELATIONSHIP WITH BIOSOCIODEMOGRAPHIC \\ CHARACTERISTICS IN HEMODIALYSED PATIENTS
}

\section{SAÚDE MENTAL E SUA RELAÇÃO COM AS CARACTERÍSTICAS BIOSOCIODEMOGRÁFICAS EM PACIENTES HEMODIADOS}

\author{
Magali Rodríguez Vidal \\ Universidad de Concepción, Concepción Chile. \\ Magaestela12@gmail.com \\ ORCID: 0000-0001-9595-121X
}

Elizabeth Badilla Aguilera

Centro de Salud Talcahuano, Chile

ORCID: 0000-0002-9887-0818

\section{Mónica Cruz Pedreros \\ Universidad de Concepción, Concepción Chile \\ ORCID: 0000-0003-1562-8063}

DOI: https://doi.org/10.22235/ech.v8i1.1801

Recibido: $13 / 11 / 2018$

Aceptado:05/04/2019

\begin{abstract}
RESUMEN
La Enfermedad renal crónica terminal es considerada una pandemia a nivel mundial. En Chile un $32 \%$ de los recursos económicos en salud son destinados a su tratamiento, siendo la hemodiálisis la terapia más utilizada en estos usuarios. Esta patología modifica todo el equilibrio de la persona, sometiéndola a diversos factores estresantes y provocándole alteraciones en el ámbito físico, psicológico y social, repercutiendo directamente en la salud mental de estos usuarios. El objetivo de esta investigación fue identificar la relación de la salud mental con las características biosociodemográficas de los pacientes hemodializados en una unidad de cuidado en Talcahuano, Chile. Se realizó un estudio cuantitativo descriptivo, correlacional y transversal. Los datos se recolectaron a través de una entrevista. El instrumento utilizado para medir salud mental fue la Escala de Golberg, más variables biosociodemográficas. Los resultados obtenidos mostraron que de un total de 53 pacientes, un 58,5\% presentó ausencia de psicopatología, 26,4\% presencia y el $15,1 \%$ sospecha; un $56,6 \%$ eran varones, el $44,4 \%$ mujeres, un $62,3 \%$ se encontraba en pareja, un $50,9 \%$ poseía estudios de nivel medio, el $83 \%$ se encontraban pensionado; la media del tiempo en hemodiálisis fue de 94,2 meses. La presencia de diabetes mellitus se relacionó con una mayor incidencia de sospecha psicopatológica. Este estudió permitió concluir que la sospecha y/o presencia de psicopatología se relacionó con edad avanzada, sexo femenino, ingresos económicos bajos, mayor tiempo en hemodiálisis, estar pensionado y padecer diabetes. La ausencia se relacionó con mayor nivel educacional y tener trabajo remunerado.
\end{abstract}

Palabras claves: salud mental, hemodiálisis, enfermería, características humanas. 


\begin{abstract}
End-stage renal disease is considered a pandemic worldwide. In Chile $32 \%$ of the economic resources in health are destined to its treatment, hemodialisys being the most common treatment in those patients. This pathology modifies the entire balance of the person, subjecting it to stress and causing alterations in their physical, psychological and social behavior, directly impacting in the mental health of patients. The objective of this research was to identify the relationship between mental health and the biosociodemographic characteristics in hemodialysis patients, from the Chronic Unit at Hospital in Talcahuano. A descriptive, correlational and transversal quantitative study was carried out. The data was collected through an interview. The instrument used to measure mental health was the Golberg Scale, plus other biosociodemographic variables. Out of 53 participants, 58.5\% presented absence of psychopathology, 26.4\% presence and 15.1\% suspicion. There were $56.6 \%$ men and $44.4 \%$ women, $60.3 \%$ were in a relationship, 50.9\% had medium level formal studies, $83 \%$ were pensioned, and the average time on hemodialysis was 94.2 months. It was also found that the presence of diabetes mellitus was associated with a higher incidence of the suspected psychopathology. Therefore, this study allowed us to conclude that the suspicion and / or presence of psychopathology was related to advanced age, female gender, low economic income, longer time on hemodialysis, being pensioned and suffering from diabetes. The absence was related to a higher educational level and having better paid jobs.
\end{abstract}

Keywords: mental health, hemodialysis, nursing, human characteristics.

\title{
RESUMO
}

A doença renal crônica terminal é considerada uma pandemia em todo o mundo. No Chile, aproximadamente $32 \%$ dos recursos econômicos em saúde são destinados ao seu tratamento, sendo a hemodiálise a terapia mais utilizada no caso desses usuários. Essa patologia modifica todo o equilíbrio da pessoa, submetendo-a a diversos fatores estressantes e provocando alterações no estado físico, psicológico e social, repercutindo diretamente na saúde mental desses usuários. $\mathrm{O}$ objetivo desta pesquisa foi identificar a saúde mental e sua relação com as características biosociodemográficas em pacientes com hemodiálise do Talcahuano, Chile. Foi realizado um estudo quantitativo, descritivo, correlacional e transversal. Os dados foram coletados por meio de entrevista. $\mathrm{O}$ instrumento utilizado foi a Escala de Golberg, para medir a saúde mental, assim como variáveis biosociodemográficas. Os resultados obtidos mostraram que: de um total de 53 pacientes, 58,5\% apresentavam ausência de psicopatologia, 26,4\% presença e $15,1 \%$ suspeita; 56,6\% homens e $44,4 \%$ mulheres, $62,3 \%$ tinham parceiro ou parceira, aproximadamente $50,9 \%$ tinham estudos de nível médio, 83\% eram aposentados, e o tempo médio em hemodiálise foi de 94,2 meses. A presença de diabetes mellitus foi associada a uma maior incidência de suspeita de psicopatologia. Este estudo permitiu concluir que a suspeita e / ou presença de psicopatologia guardava relação com a idade avançada, sexo feminino, renda econômica baixa, maior tempo em hemodiálise, estar aposentado e sofrer de diabetes. A ausência foi relacionada a um maior nível de escolaridade e trabalho remunerado.

Palavras-chave: saúde mental, hemodiálise, enfermagem, características humanas. 


\section{INTRODUCCIÓN}

En la actualidad la enfermedad renal crónica (ERC) constituye un importante problema de salud pública, cuya manifestación más avanzada es la enfermedad renal crónica terminal (ERCT), que requiere tratamiento sustitutivo de la función renal mediante diálisis o trasplante. Según estudios españoles al 2005 las causas más frecuentes de ERC corresponden en su mayoría a una mala adherencia terapéutica de enfermedades crónicas no transmisibles como la Diabetes Mellitus (DM) e Hipertensión Arterial (HTA), las que se han triplicado en las últimas décadas, constituyendo una pandemia a nivel mundial (1).

En Chile un 32\% del presupuesto al Acceso Universal de Garantías Explícitas (AUGE) en salud es destinado al tratamiento de la ERCT (2). La diálisis consiste en un método depurativo de toxinas, electrolitos y exceso de agua del organismo, que se puede ejecutar a través de dos técnicas. Una es la diálisis peritoneal, que utiliza la membrana peritoneal, un catéter que une la membrana con el exterior y líquido dializante; este procedimiento es ambulatorio y lo realiza el paciente en su hogar. El segundo es la hemodiálisis, que implica el uso de un filtro (membrana artificial) a través del cual pasa la sangre y retorna al paciente limpia; es un tratamiento invasivo, ambulatorio, que requiere tres sesiones por semana de cuatro horas por vez, realizado en centros hospitalarios o clínicas, por equipos humanos y usando materiales altamente especializados, que requiere además el uso permanente de fármacos, cuidados específicos del tratamiento dialítico, modificaciones estrictas en la dieta, y cambios de estilos de vida (3-6).

La enfermedad renal altera todo el equilibrio de la persona y su medio sometiéndola a diversos factores estresantes, provocándole múltiples alteraciones en el ámbito físico, psicológico y social. En el ámbito físico experimentan problemas cardiovasculares (hipertensión, hipotensión, angina), en músculos esqueléticos (dolor, calambres, fatiga,) gastrointestinales, (náuseas, vómitos y anorexia), inmunológicos, (alergias cutáneas) y disfunción sexual. En el ámbito psicológico, afecciones del ánimo y a nivel socioeconómico, incapacidad de poseer un trabajo a tiempo completo y con frecuencia pérdida del trabajo anterior, desencadenando problemas económicos, personales y familiares que afectan directamente la calidad de vida y la salud mental de estos usuarios $(7,8)$.

Joana Fornés en el año 2015 define salud mental como un "Estado de bienestar en el cual el individuo es consciente de sus propias capacidades, puede afrontar las tensiones normales de la vida, puede trabajar de forma productiva y fructífera y es capaz de hacer una contribución a su comunidad". Es dinámica, fluida, y cambia con los factores de estrés que surgen en la vida diaria (9). Los cambios generados por la ERCT y el tratamiento de hemodiálisis producen alteraciones en la salud mental en un porcentaje importante de pacientes. Los trastornos del ánimo y ansiedad se consideran frecuentes en los primeros seis meses de tratamiento, pero cuando se superan los seis meses resultan preocupantes para la salud mental, dando pie a la aparición de psicopatologías como depresión y trastornos de ansiedad, más frecuentes en mujeres y en edades tempranas para ambos sexos $(4,7,8,10-12)$. La depresión lidera los trastornos del ánimo, produciendo mayor morbilidad y mortalidad en estos usuarios en comparación con otros trastornos, reflejándose en la falta de adherencia terapéutica, específicamente al régimen dietético e inasistencia a los controles médicos $(13,14)$. La depresión en Chile, según la Encuesta 
Nacional 2016-2017, mostró una prevalencia de 6,2\% en la población en general, siendo mayor en mujeres, con una incidencia de $10,1 \%$, que en hombres $(2,1 \%)(15)$. La ansiedad posee una incidencia de $17,9 \%$ en la población general (16). Estos datos permiten deducir que las patologías de salud mental se encuentran presentes en un gran número de la población chilena. Los trastornos en la salud mental generan un fenómeno llamado AVISA, que consiste en la pérdida de años de vida saludable por causa de una patología. La depresión es la primera causa de AVISA en mujeres y afecta a un 23,2\% de la población en general (14). Según la Sociedad Chilena de Nefrología en el año 2017 la mortalidad bruta de estos pacientes fue del 10,9\%, y de éstos, 128 abandonaron el tratamiento por decisión propia o de la familia, sin especificar las causas del abandono o antecedentes de psicopatología (2).

Frente a lo expuesto, para Enfermería como gestor del cuidado es relevante identificar el nivel de salud mental de estos usuarios, pesquisando de forma precoz alteraciones precursoras de fenómenos tales como baja adherencia terapéutica y abandono de tratamiento, entre otros, considerando factores protectores de cada persona, y así generar estrategias en pro de mejorar su salud mental, evitando hechos desafortunados en este tipo de paciente. El siguiente estudio pretendió identificar el nivel de salud mental y su relación con las características biosociodemográficas de los pacientes hemodializados de una Unidad de Crónicos en las Higueras, Talcahuano, durante el periodo febrero-marzo 2017.

\section{METODOLOGÍA}

Se realizó un estudio cuantitativo descriptivo, transversal y correlacional a pacientes en tratamiento con hemodiálisis crónica, durante el primer semestre del año 2017 en una Unidad de Diálisis de un Hospital en las Higueras, comuna de Talcahuano, Chile. En cuanto a la población y muestra, para efectos estadísticos, se trabajó con la totalidad de la población de 53 personas. El criterio de inclusión fue estar en tratamiento de hemodiálisis crónica en ese centro y que accedieran a participar voluntariamente, mientras que los de exclusión fueron que los pacientes crónicos fueran de otros centros, pacientes de la unidad de agudos y aquellos que se negaron a participar. El procedimiento de recolección de datos se realizó a través de una entrevista verbal realizada por una de las autoras durante las primeras dos horas de terapia dialítica.

El instrumento de medida utilizado fue el instrumento recolector "Perfil biopsicosocial de los pacientes hemodializados", que constó de dos secciones: la primera, un cuestionario de preguntas biosociodemográficas (edad, sexo, ingreso económico per cápita, patologías agregadas, consumo de alcohol, estado civil, situación laboral, residencia, tiempo en hemodiálisis, escolaridad y cantidad de personas con quien vive). La segunda, Escala de Salud de Golberg para evaluar salud mental, validada al español y aprobada por el Ministerio de Salud de Chile, Trucco y Cols. citado por Guic Sa et al en el año 2006. La describen como de alta confiabilidad y validez; consta de doce preguntas que evalúan la presencia de trastornos psiquiátricos: depresión, ansiedad, inadecuación social e hipocondría; otorga cuatro opciones de respuesta en formato tipo Likert con un mínimo de 0 puntos a un máximo de 12 puntos, dividido en tres categorías: ausencia de psicopatología (0 a 4 ptos), sospecha de patología subumbral (5 a 6 ptos) y presencia de psicopatología ( 7 a 12 ptos =indicativo) (17). El estudio contó con la aprobación del comité de Ética de la Facultad de Enfermería de la Universidad de Concepción y el comité de investigación del Hospital, además del consentimiento informado y firmado por cada 
uno de los participantes, considerando y respetando los siete requisitos éticos planteados por Ezekiel Emanuel.

Para el análisis estadístico, los datos obtenidos se volcaron a una planilla Excel versión 2010, y fueron analizados en el software SPSS V. 17, patentado en la Universidad de Concepción. Para la estadística descriptiva se empleó distribución de frecuencias, medidas de tendencia central y medidas de dispersión (rangos y desviación estándar). Para el análisis inferencial se utilizó prueba Chi-Cuadrado de independencia y correlación de Pearson.

\section{RESULTADOS}

El perfil biosociodemográfico de la población estuvo constituida por 53 pacientes, predominando los hombres $(56,6 \%)$ sobre las mujeres. El promedio de edad correspondió a 58,9 años (18 - 87 años), más de la mitad estaba casado o en pareja (62,3\%), y un $11,3 \%$ vivían solos. Un 50,9\% reportó estudios de nivel medio, 28,3\% estudios básicos y $17 \%$ superiores. En relación a la situación laboral, la mayoría de ellos (83\%) se encontraba pensionado y sólo un $17 \%$ trabajaba o estudiaba. Un $98,1 \%$ contaba con saneamiento básico y habitaba en el sector urbano, y un 96,2\% refirió no tener hábito de consumo de alcohol (Tablas 1 y 2).

Tabla 1. Distribución nominal y porcentual de las características biosociodemográficas de pacientes hemodializados.

\begin{tabular}{|c|c|c|c|}
\hline VARIABLES & CATEGORIA & FRECUENCIA & PORCENTAJE \\
\hline \multirow{2}{*}{ SEXO } & Masculino & 30 & 56,6 \\
\hline & Femenino & 23 & 43,4 \\
\hline \multirow{2}{*}{ ESTADO CIVIL } & Con pareja & 33 & 62,3 \\
\hline & Sin pareja & 20 & 37,7 \\
\hline \multirow{4}{*}{ ESCOLARIDAD } & Ninguna & 2 & 3,9 \\
\hline & Básica & 15 & 28,3 \\
\hline & Media & 27 & 50,9 \\
\hline & Superior & 9 & 17,0 \\
\hline \multirow{2}{*}{$\begin{array}{l}\text { SITUACION } \\
\text { LABORAL }\end{array}$} & Pensionado & 44 & 83,0 \\
\hline & $\begin{array}{l}\text { Trabaja } \\
\text { estudia }\end{array}$ & 9 & 17,0 \\
\hline \multirow{2}{*}{ RESIDENCIA } & Urbana & 52 & 98,1 \\
\hline & Rural & 1 & 1,9 \\
\hline \multirow{2}{*}{$\begin{array}{l}\text { CONSUMO DE } \\
\text { ALCOHOL }\end{array}$} & No & 51 & 96,2 \\
\hline & $\mathrm{Si}$ & 2 & 3,8 \\
\hline
\end{tabular}

Fuente: Elaboración propia (2017) 
En la Tabla 2 podemos observar las medias de las variables cuantitativas. El tiempo en hemodiálisis fue de 94,2 meses, el ingreso per cápita mensual fluctuó entre U\$S31.4 y U\$S1007, el número de personas que habitaban en el hogar presentó un rango de 0 a 11 , con una media de 2,55 y de las patologías agregadas se encontró que la diabetes $(50,9 \%)$ y la hipertensión $(83 \%)$ predominaron sobre las otras enfermedades crónicas.

Tabla 2: Segunda parte de la distribución nominal y porcentual de las características

\begin{tabular}{|c|c|c|c|c|}
\hline & VARIABLES BIOSOCIODEMOGRAFICAS CUANTITATIVAS & Media \\
\hline & $\mathrm{N}$ & Mínimo & Máximo & 58,9 \\
\hline EDAD & 53 & 18 & 87 & 94,2 \\
\hline INGMPO EN HD & 53 & 3 & 393 & US\$233,87 \\
\hline PERCAPITA & 53 & US $\$ 31,4$ & US\$1007 & 2,55 \\
\hline $\begin{array}{c}\text { CANTIDAD DE } \\
\text { PERSONAS CON }\end{array}$ & 53 & 0 & 11 & \\
\hline QUIEN VIVEN & & & & \\
\hline
\end{tabular}

Fuente: Elaboración propia (2017)

Tabla 3: Salud Mental según la Escala de Golberg en los pacientes sometidos a hemodiálisis en una Unidad de Crónicos de un Hospital en Las Higueras Talcahuano, 2017.

\begin{tabular}{|c|c|c|c|}
\hline \multirow{4}{*}{ SALUD MENTAL } & Nivel & Frecuencia & Porcentaje \\
\hline & $\begin{array}{ll}\text { Ausencia } & \text { de } \\
\text { psicopatología } & \end{array}$ & 31 & 58,5 \\
\hline & $\begin{array}{ll}\text { Sospecha } & \text { de } \\
\text { psicopatología } & \end{array}$ & 8 & 15,1 \\
\hline & $\begin{array}{ll}\text { Presencia } & \text { de } \\
\text { psicopatología } & \end{array}$ & 14 & 26,4 \\
\hline
\end{tabular}

Fuente: Elaboración propia (2017)

Respecto a la salud mental valorada por la Escala de Golberg (Tabla 3) se encontró que un $58,5 \%$ presentó ausencia de psicopatología, un 26,4\% presencia y el 15,1\% sospecha de ella. Del análisis de algunos ítems se reportó que un $83 \%$ no presentó alteración en la toma de decisiones, un $81,1 \%$ manifestó encontrarse feliz pese a sus circunstancias y un $77,4 \%$ refirió haber logrado hacer frente a sus dificultades pese a ellas, un 52,8\% presentó alteración de la percepción del papel que desarrollan en la vida, el 59,9\% mencionó que la calidad del sueño ha sido afectada por sus preocupaciones, y el 45,3\% se ha sentido deprimido o decaído en el último tiempo y en igual proporción agobiados y en tensión. (Gráficos 1 y 2) 
Gráfico 1: Tendencia a la ausencia de psicopatología en los pacientes sometidos a hemodiálisis

\begin{tabular}{|l|c|}
\hline ¿Se ha sentido capaz de tomar decisiones? & $83,0 \%$ \\
\hline ¿Se siente razonablemente feliz considerando todas sus circunstancias? & $81,1 \%$ \\
\hline ¿Ha sido capaz de hacer frente adecuadamente a sus problemas? & $77,4 \%$ \\
\hline ¿Ha sentido que no puede superar sus dificultades? & $71,7 \%$ \\
\hline ¿Ha perdido la confianza en sí mismo/a? & $67,9 \%$ \\
\hline ¿Ha pensado que es usted una persona que no vale para nada? & $66,0 \%$ \\
\hline ¿Ha sido capaz de disfrutar de sus actividades normales de cada día & $66,0 \%$ \\
\hline ¿Ha podido concentrarse bien en lo que hace? & $62,3 \%$ \\
\hline ¿Se ha sentido poco feliz o deprimido? & $54,7 \%$ \\
\hline ¿Se ha sentido constantemente agobiado y en tensión? & $54,7 \%$ \\
\hline ¿Sus preocupaciones le han hecho perder mucho el sueño? & $49,1 \%$ \\
\hline ¿Ha sentido que está desempleando un papel útil en la vida? & $47,2 \%$ \\
\hline
\end{tabular}

Fuente: Elaboración propia (2017)

Grafico 2: Tendencia a la presencia y sospecha de psicopatología en los pacientes sometidos a hemodiálisis

\begin{tabular}{|l|l|}
\hline ¿Ha sentido que está desempleando un papel útil en la vida? & $52,8 \%$ \\
\hline ¿Sus preocupaciones le han hecho perder mucho el sueño? & $50,9 \%$ \\
\hline ¿Se ha sentido poco feliz o deprimido/a? & $45,3 \%$ \\
\hline ¿Se ha sentido constantemente agobiado/a o en tensión? & $45,3 \%$ \\
\hline ¿Ha podido concentrarse bien en lo que hace? & $37,7 \%$ \\
\hline ¿Ha pensando que usted es una persona que no vale para nada? & $34,0 \%$ \\
\hline ¿Ha sido capaz de disfrutar de sus actividades normales de cada día? & $34,0 \%$ \\
\hline ¿Ha perdido la confianza en sí mismo/a? & $32,1 \%$ \\
\hline ¿Ha sentido que no puede superar sus dificultades? & $28,3 \%$ \\
\hline ¿Ha sido capaz de hacer frente adecuadamente a sus problemas? & $22,6 \%$ \\
\hline ¿Se siente razonablemente feliz considerando todas sus circunstancias? & $18,9 \%$ \\
\hline ¿Se ha sentido capaz de tomar decisiones? & $17,0 \%$ \\
\hline
\end{tabular}

Fuente: Elaboración propia (2017) 
Del análisis bivariado se pudo deducir que el sexo femenino poseía mayor tendencia a presentar trastornos de salud mental $\left(\mathrm{chi}^{2} 0,328\right)$; la edad presentó una correlación de Pearson de $+0,115$, deduciendo que a mayor edad existe una mayor probabilidad de presentar alteraciones en la salud mental; quienes tenían actividad laboral o estudiantil $\left(\mathrm{chi}^{2} 0,116\right)$ y mayor nivel de escolaridad $\left(\mathrm{chi}^{2} 0,141\right)$ presentaron mayor probabilidad de ausencia de psicopatología, (tabla 4).

Tabla 4: Relación entre el nivel de salud mental y el sexo de los pacientes hemodializados en una Unidad de Crónicos de un Hospital en Las Higueras, Talcahuano

\begin{tabular}{|c|c|c|c|c|c|}
\hline \multirow{2}{*}{\multicolumn{2}{|c|}{$\begin{array}{l}\text { Variables } \\
\text { biosociodemograficas }\end{array}$}} & \multicolumn{4}{|c|}{ NIVEL DE SALUD MENTAL } \\
\hline & & & $\begin{array}{l}\text { Ausencia de } \\
\text { psicopatología }\end{array}$ & $\begin{array}{l}\text { Sospecha de } \\
\text { psicopatología }\end{array}$ & $\begin{array}{l}\text { Presencia de } \\
\text { psicopatología }\end{array}$ \\
\hline \multirow{4}{*}{ Sexo } & \multirow{4}{*}{ Masculino } & Frecuencia & 20 & 3 & 7 \\
\hline & & $\begin{array}{l}\text { Porcentaje } \\
\text { dentro del } \\
\text { sexo }\end{array}$ & 66,7 & 10,0 & 23,3 \\
\hline & & Frecuencia & 11 & 5 & 7 \\
\hline & & $\begin{array}{l}\text { Porcentaje } \\
\text { dentro del } \\
\text { sexo }\end{array}$ & 47,8 & 21,7 & 30,4 \\
\hline \multirow{4}{*}{$\begin{array}{l}\text { Estado civil } \\
\text { E. C }\end{array}$} & \multirow[b]{2}{*}{ Con pareja } & Frecuencia & 19 & 6 & 8 \\
\hline & & $\begin{array}{l}\text { Porcentaje } \\
\text { dentro E.C }\end{array}$ & 57,6 & 18,2 & 24,2 \\
\hline & \multirow[b]{2}{*}{ Sin pareja } & Frecuencia & 12 & 2 & 6 \\
\hline & & $\begin{array}{l}\text { Porcentaje } \\
\text { dentro EC }\end{array}$ & 60 & 10 & 30 \\
\hline \multirow{8}{*}{$\begin{array}{l}\text { Escolaridad } \\
\text { (E) }\end{array}$} & \multirow[t]{2}{*}{ Ninguna } & Frecuencia & 0 & 1 & 1 \\
\hline & & $\begin{array}{l}\text { Porcentaje } \\
\text { dentro E. }\end{array}$ & 0 & 50 & 50 \\
\hline & \multirow[t]{2}{*}{$\begin{array}{l}\text { Enseñanza } \\
\text { básica }\end{array}$} & Frecuencia & 6 & 4 & 5 \\
\hline & & $\begin{array}{l}\text { Porcentaje } \\
\text { dentro E. }\end{array}$ & 40 & 26,7 & 33,3 \\
\hline & \multirow[t]{2}{*}{$\begin{array}{l}\text { Enseñanza } \\
\text { media }\end{array}$} & Frecuencia & 17 & 3 & 7 \\
\hline & & $\begin{array}{l}\text { Porcentaje } \\
\text { dentro E. }\end{array}$ & 63,3 & 11,1 & 25,9 \\
\hline & \multirow[t]{2}{*}{$\begin{array}{l}\text { Enseñanza } \\
\text { superior }\end{array}$} & Frecuencia & 8 & 0 & 1 \\
\hline & & $\begin{array}{l}\text { Porcentaje } \\
\text { dentro E. }\end{array}$ & 88,9 & 0 & 11,1 \\
\hline
\end{tabular}

Fuente: Elaboración propia (2017) 
En relación al número personas con quien vive el paciente se encontró que, a mayor número, mayor era la probabilidad de sospecha o presencia de psicopatología (correlación de Pearson de 0,12). Las personas solas presentaron mayor tendencia a ausencia de psicopatología ( $\mathrm{chi}^{2}$ 0.7). Respecto a los ingresos per cápita se encontró que, a mayores ingresos, mayor ausencia de psicopatología (Pearson 0,112) y a mayor tiempo en tratamiento dialítico, mayor posibilidad de alteración en la salud mental (Pearson 0,143). En relación a patologías agregadas, sólo los usuarios portadores de diabetes mellitus tuvieron mayor tendencia a presentar sospecha y/o presencia de psicopatología $\left(\mathrm{chi}^{2} 0,04\right)$ (Ver Tabla 5)

Tabla 5: Relación entre el nivel de salud mental y actividad en los pacientes hemodializados.

\begin{tabular}{|c|c|c|c|c|c|}
\hline \multirow{2}{*}{\multicolumn{2}{|c|}{$\begin{array}{l}\text { Variables } \\
\text { biosociodemograficas }\end{array}$}} & \multicolumn{4}{|c|}{ NIVEL DE SALUD MENTAL } \\
\hline & & & $\begin{array}{l}\text { Ausencia de } \\
\text { psicopatología }\end{array}$ & $\begin{array}{l}\text { Sospecha de } \\
\text { psicopatología }\end{array}$ & $\begin{array}{l}\text { Presencia de } \\
\text { psicopatología }\end{array}$ \\
\hline \multirow{4}{*}{ Actividad } & \multirow[b]{2}{*}{$\begin{array}{l}\text { Trabaja o } \\
\text { estudia }\end{array}$} & Frecuencia & 8 & 0 & 1 \\
\hline & & $\begin{array}{l}\text { Porcentaje } \\
\text { dentro de } \\
\text { actividad }\end{array}$ & 88,9 & 0 & 11,9 \\
\hline & & Frecuencia & 23 & 8 & 13 \\
\hline & Pensionado & $\begin{array}{l}\text { Porcentaje } \\
\text { dentro de } \\
\text { actividad }\end{array}$ & 52,3 & 18,2 & 29,5 \\
\hline \multirow{4}{*}{ Patologías } & \multirow{2}{*}{$\begin{array}{l}\text { Si padece } \\
\text { diabetes } \\
\text { mellitus } \\
\text { D.M }\end{array}$} & Frecuencia & 10 & 7 & 10 \\
\hline & & $\begin{array}{l}\text { Porcentaje } \\
\text { dentro de } \\
\text { DM }\end{array}$ & 37 & 26 & 37 \\
\hline & \multirow[t]{2}{*}{$\begin{array}{l}\text { No padece } \\
\text { diabetes } \\
\text { mellitus } \\
\text { D.M }\end{array}$} & Frecuencia & 21 & 1 & 4 \\
\hline & & $\begin{array}{l}\text { Porcentaje } \\
\text { dentro de } \\
\text { DM }\end{array}$ & 80,8 & 3,8 & 15,4 \\
\hline
\end{tabular}

Fuente: Elaboración propia (2017) 


\section{DISCUSION}

La población estuvo compuesta principalmente por hombres. Este fenómeno puede estar relacionado a que el sexo femenino presenta mayor adherencia terapéutica a los controles de salud e indicaciones del tratamiento (medicación, dieta, actividad, etc). La edad se presentó en un rango de 18 a 87 años, con una media de 58,9 años, similar a lo reportado a nivel nacional, donde esta patología y sus implicancias comienzan cada vez a edades más tempranas $(8,18)$.

En este estudio un número importante de la población $(58,5 \%)$ se encontró libre de psicopatologías según la Escala de Golberg. Es relevante destacar que ésta no otorga un diagnóstico específico de salud mental, sólo genera sospecha de psicopatología, la que debe ser confirmada por un especialista. La suma de sospecha más presencia representó un $41,5 \%$, que, de ser confirmado como depresión o ansiedad, presentaría similitud a lo informado por otros autores a nivel nacional e internacional $(7,8)$. Estos resultados difieren de otros estudios, que reportaron un mayor predominio de alteraciones de la salud mental, liderando síntomas depresivos, depresión severa y/o ansiedad grave en estos pacientes (4, $11,14,19)$. Esta diferencia podría deberse a causas multifactoriales, como instrumentos de medida, número de personas, sexo, tiempo en terapia dialítica, entre otras. La sospecha y presencia de psicopatología se relacionó mayoritariamente con el sexo femenino, lo que podría asociarse a características propias del género, como mayor exposición o mayor respuesta frente al estrés cotidiano, concentraciones más altas de aminas biogénicas y cambios endócrinos que se producen con la menstruación y en la menopausia, sumado al rol que posee la mujer dentro de la sociedad chilena como dueña de casa, madre, trabajadora y en algunas ocasiones jefa de hogar y/o cuidadora informal de un tercero dependiente, las cuales alterarían la capacidad de respuesta a situaciones estresantes evitando la adaptación a agentes nocivos para la salud mental, similar a lo reportado por otros autores $(10,11,14,20)$.

La edad avanzada y un mayor tiempo en hemodiálisis se relacionó afirmativamente con la presencia y sospecha de psicopatología. El envejecimiento suele ir acompañado de diversas comorbilidades y estigmas sociales que favorecen situaciones agobiantes, dando pie a la aparición de sentimientos negativos como nostalgia, melancolía y tristeza, que al perdurar en el tiempo desencadenan psicopatologías como los trastornos de ansiedad y depresión, concordante a lo reportado por otros autores $(4,10)$. Un mayor tiempo en terapia dialítica genera un proceso de transición y adaptación a los distintos agentes estresantes, que en etapas iniciales son enfrentadas adecuadamente por los usuarios, pero pasado los cinco a diez años producen un aumento de comorbilidades, disminuyendo la funcionalidad de los individuos y el desempeño en su rol social pasado, afectando su salud mental $(4,11,21)$. Llamó la atención que en este estudio el estar sin pareja no constituyó un factor de riesgo en la salud mental, pese a que las referencias empíricas nos demuestran que la compañía y el vínculo afectivo otorgado por un tercero disminuye los sentimientos de soledad y angustia, proporcionándole apoyo al paciente y al equipo de salud, facilitando el camino a la adherencia terapéutica y a la compensación de la patología (21).

El cese laboral anticipado, ingresos económicos bajos y un mayor número de personas dentro de la vivienda se relacionaron con la sospecha y presencia de psicopatología. Los primeros generarían sentimientos negativos, precursores de depresión, específicamente en aquellos usuarios en edad productiva de la vida y jefes/as de hogar. Los segundos pueden 
deberse a condiciones de hacinamiento que dificultarían las relaciones interpersonales, aumentando los agentes estresantes dentro del contexto familiar $(4,11)$.

En el ámbito cognitivo y educacional se encontró que una mayor escolaridad se relacionaba positivamente con la ausencia de psicopatología. Un mayor desarrollo intelectual pudiese facilitar la adaptación y comprensión a los cambios abruptos que conlleva la ERCT y sus implicancias negativas, favoreciendo actitudes de autocuidado y afrontamiento eficaz, con el fin de retrasar las complicaciones propias de este tratamiento. A la luz de estos resultados los equipos de salud deben facilitar la asimilación a estos cambios, adecuando sus intervenciones educativas en aquellos pacientes con menor escolaridad con el fin de disminuir la brecha entre ambos grupos (11).

La mayoría de estos usuarios reportó no consumir alcohol (96\%), siendo éste un factor protector de la salud mental, ya que la literatura informa que el consumo activo de alcohol se relaciona con la ideación suicida en estos pacientes, reflejando una mala adherencia terapéutica y una disminución de su autocuidado. Este hábito puede ser un método de afrontamiento ineficaz a las diversas comorbilidades producidas por la patología y su tratamiento, y a la vez generar un incremento de éstas, desencadenando un proceso de simbiosis entre ambos sucesos (22).

En este estudio los pacientes diabéticos en tratamiento de sustitución renal demostraron una relación positiva con la sospecha y presencia de psicopatologías, probablemente originadas por la sumatoria de comorbilidades y deterioro progresivo multisistémico, que favorecerían la presencia de alteraciones en las relaciones familiares, sociales y laborales afectando la calidad de vida y la salud mental de estos usuarios. La presencia de diabetes por sí misma no conlleva necesariamente a una afección negativa en la salud mental de los individuos, aunque los múltiples cambios en el estilo de vida producidos por esta patología podrían ser un factor premonitor de deterioro en la salud mental de estos sujetos $(7,8)$.

\section{CONCLUSIONES Y CONSIDERACIONES FINALES}

La población de los pacientes hemodializados de la Unidad de Crónicos del Hospital en Talcahuano posee una mayor tendencia a la ausencia de psicopatología. Una salud mental óptima se relacionó con ingresos económicos altos, menor edad, sexo masculino, realizar alguna actividad (trabajar o estudiar), vivir acompañado, niveles educacionales altos, menor tiempo en hemodiálisis y menor número de personas con las que convivían. La sospecha y presencia de psicopatología se relacionó con el sexo femenino, edad avanzada, ingresos económicos y nivel educacional bajos, ser pensionado, padecer diabetes, llevar más tiempo en terapia dialítica y convivir con un mayor número de personas en el hogar.

En base a lo expuesto, es responsabilidad de los equipos de salud idear estrategias y protocolos específicos para detectar afecciones tempranas en la salud mental en esta población, otorgando un cuidado holístico, humanizado y multidisciplinar, desarrollando intervenciones que favorezcan su propio autocuidado, independencia y autoestima, disminuyendo sentimientos de angustia, tristeza y dependencia entre otros. En el desarrollo de estas estrategias es relevante destacar la función del profesional de enfermería, quien es el responsable del cuidado directo y permanente de estos usuarios, por lo que sería recomendable implementar, dentro del proceso enfermero, una valoración específica y 
programada de salud mental, con instrumentos validados que faciliten la detección precoz permitiendo un manejo oportuno a través de una intervención en crisis y derivación oportuna en caso necesario.

\section{REFERENCIAS BIBLIOGRAFICAS}

1. Ulloa Varela J, Rodríguez Vidal M, Cruz Pedreros M. Perfil biosociodemográfico y valoración de necesidades en pacientes en diálisis peritoneal. Cienc. enferm. [Internet]. 2014 Abr [citado 2018 Nov 2] ; 20 ( 1 ): 89-95. Disponible en:

https://revistas.ucu.edu.uy/index.php/enfermeriacuidadoshumanizados/about/submis sions\#authorGuidelines

2. Sociedad Chilena de Nefrología. Poblete Badal H. www.nefro.cl. [Online].; (31 agos 2017) [cited 2018 octubre 8] Available from: https://www.nefro.cl/v2/biblio/registro/23.pdf.

3. Sociedad Argentina de Cardiología.Veltri M Insuficiencia renal crónica: ¿qué debemos conocer para el manejo cotidiano. PROSAC.( Internet) 2011 (citado 2 oct 2018) ; 7(2) disponible en:

http://educacion.sac.org.ar/pluginfile.php/2972/mod_page/content/2/1_Dr. Veltri.pdf

4. Páez AE, Jofré MJ, Azpiricoz CR, Bortoli MAD. Ansiedad y depresión en pacientes con insuficiencia renal crónica terminal en tratamiento de diálisis. Universitas Psychologica( Internet) (2009); (citado Oct 3 2018) 8 (1) 117-124. Disponible en: http://www.redalyc.org/articulo.oa?id=64712168010

5. Mariné AlonsoM, García Melián M. Lista de verificación para la identificación de peligros ambientales en unidades de hemodiálisis. Rev Cubana Hig Epidemiol [Internet]. 2010 Abr [citado 2018 Nov 5] ; 48( 1 ). Disponible en: http://scielo.sld.cu/scielo.php?script=sci_arttext\&pid=S1561-30032010000100005

6. Daugirdas JT. Manual de diálisis. 4th ed. barcelona : lippincott williams and wilkins. wolters kluwer health; 2015.

7. Perales-Montilla C, Duschek S, Reyes-del Paso G.. Influencia de los factores emocionales sobre el informe de síntomas somáticos en pacientes en hemodiálisis crónica: relevancia de la ansiedad. Nefrología (Madr.) [Internet]. 2013 [citado 2018 Oct 3] ; 33( 6 ): 816-825. Disponible en: http://scielo.isciii.es/scielo.php?script=sci_arttext\&pid=S0211-69952013000700009

8. Arechabala MC, Catoni MI, Palma E, Barrios S. Depresión y auto percepción de la carga del cuidado en pacientes en hemodiálisis crónica y sus cuidadores. Rev Panam Salud Pública(Internet) 2011. Citado oct 5 2018;30(1):74-9. Disponible en: https://scielosp.org/pdf/rpsp/2011.v30n1/74-79/es

9. Vives JF. Enfermería de Salud Mental y Psiquiátrica. Valoración y cuidados. segunda edición ed. Fornes: Editoria Medica Panamericana.; 2011.

10. Gómez Villaseca L, Manresa Traguany M, Pac Gracia N, Lozano Ramírez S. Prevalencia de ansiedad y depresión en pacientes de hemodiálisis. Enferm Nefrol [Internet]. 2014 [citado 2018 Oct 5] ; 17( Suppl 1 ): 26-26. Disponible en: http://scielo.isciii.es/scielo.php?script=sci_arttext\&pid=S2254-28842014000500015

11. Capote Leyva E, Torres Lio-Coo V. Prevalencia y comportamiento clínico de síntomas depresivos en pacientes en hemodiálisis periódica intermitente. Rev Cub Med Mil [Internet]. 2014 Mar [citado 2018 oct 5] ; 43( 1 ): 61-71. Disponible en: 
http://scielo.sld.cu/scielo.php?script=sci_arttext\&pid=S0138-65572014000100008

12. Sánchez Lamolda M, Malagón Rodríguez M, Alonso Hernández N. Incidencia de las demencias en hemodiálisis: Apoyo al cuidador principal. Enferm Nefrol [Internet]. 2013 Mar [citado 2018 Oct 5] ; 16( 1 ): 31-35. Disponible en: http://scielo.isciii.es/scielo.php?script=sci_arttext\&pid=S2254-28842013000100005

13. Cukor, D et al. Anxiety Disorders in Adults Treated by Hemodialysis: A SingleCenter Study American Journal of Kidney Diseases , ( Internet) (citado en 2018 oct 5) $52,1,128$ - 136 disponible en:

https://www.ajkd.org/article/S0272-6386(08)00530-1/fulltext

14. Rojas-Villegas Y, Ruíz-Martínez A, \& González-Sotomayor R. Ansiedad y depresión sobre la adherencia terapéutica en pacientes con enfermedad renal. Revista de psicología (Santiago) (internet) (2017) citado 2018 Oct 5., 26(1), 65-77. Disponible en:

https://scielo.conicyt.cl/scielo.php?script=sci_arttext\&pid=S071905812017000100065

15. Ministerio de Salud de Chile. Encuesta Nacional de Salud 2016-2017 Minsal. [Internet].; 2017 (cited 2018 octubre 22). Disponible en: https://www.cooperativa.cl/noticias/site/artic/20180131/asocfile/20180131233128/2 resultados_ens_minsal_31_01_2018.pdf.

16. Ministerio de Salud de Chile. Guía Clínica Depresión en personas de 15 años y más. (internet) MINSAL, Santiago $2^{\mathrm{a}}$ edición 2013. Disponible en: https://www.minsal.cl/portal/url/item/7222754637c08646e04001011f014e64.pdf

17. Guic S E, Mora O, Rey R, Robles A. Estrés organizacional y salud en funcionarios de centros de atención primaria de una comuna de Santiago. Rev. méd. Chile [Internet]. 2006 Abr [citado 2018 Oct 11] ; 134( 4 ): 447-455. Disponible en:

https://scielo.conicyt.cl/scielo.php?script=sci_arttext\&pid=S003498872006000400007

18. Veliz-Rojas L, Mendoza-Parra S, Barriga Omar A. Adherencia terapéutica en usuarios de un programa de salud cardiovascular de atención primaria en Chile. Rev. perú. med. exp. salud publica [Internet]. 2015 Ene [citado 2018 Oct 20] ; 32( 1 ): 51-57. Disponible en:

http://www.scielo.org.pe/scielo.php?script=sci_arttext\&pid=S172646342015000100008

19. Arribas Cobo P, García Estévez S, Díaz de Argote Cervera P, Quesada Armenteros M, Liébana Pamos B, et al. Prevalencia de fatiga postdiálisis, depresión y dolor del paciente en hemodiálisis. Enferm Nefrol [Internet]. 2014 [citado 2018 Oct 21] ; 17( Suppl 1 ): 27-27. Disponible en:

http://scielo.isciii.es/scielo.php?script=sci arttext\&pid=S2254-28842014000500016

20. Villanueva Peña, C; Casas Caruajulca, J, Richard Pérez S. Depresión y calidad de vida en pacientes con tratamiento de hemodiálisis en un Hospital De EsSalud en Lima - Perú, 2014 Revista de Investigación Universitaria, ( internet) 2015, citado el 2018 Oct 20) Vol. 4 (1): 42-50. Disponible en: https://revistas.upeu.edu.pe/index.php/riu/article/view/666/637

21. Mesa BM, Vázquez AY, Álvarez MNR. Depresión y ansiedad en los pacientes de hemodiálisis, Instituto de Nefrología, 2011-2012. Revista del Hospital Psiquiátrico de La Habana ( Internet) 2013. Citado 2018 oct 22. 10 (3). Disponible en: http://www.medigraphic.com/pdfs/revhospsihab/hph-2013/hph133e.pdf 
22. Martinez Bertolaccini B, Custodio Rodrigo Pereira R. Relationship between mental health and spiritual wellbeing among hemodialysis patients: a correlation study. Sao Paulo Med. J. [Internet]. 2014 [citado 2018 Oct 22] ; 132( 1 ): 23-27. Disponible en:

http://www.scielo.br/scielo.php?script=sci_arttext\&pid=S1516$31802014000100023 \& \operatorname{lng}=$ en\&nrm=iso\&tlng=en 